\title{
Emotional Inertia Prospectively Predicts the Onset of Depressive Disorder in Adolescence
}

\author{
Peter Kuppens \\ University of Leuven and University of Melbourne
}

\author{
Lisa B. Sheeber \\ Oregon Research Institute, Eugene, OR
}

\begin{abstract}
Marie B. H. Yap, Sarah Whittle, Julian G. Simmons, and Nicholas B. Allen
University of Melbourne and Orygen Youth Health Research Centre, Melbourne, Australia

Emotional inertia refers to the degree to which a person's current emotional state is predicted by their prior emotional state, reflecting how much it carries over from one moment to the next. Recently, in a cross-sectional study, we showed that high inertia is an important characteristic of the emotion dynamics observed in psychological maladjustment such as depression. In the present study, we examined whether emotional inertia prospectively predicts the onset of first-episode depression during adolescence. Emotional inertia was assessed in a sample of early adolescents $(N=165)$ based on second-to-second behavioral coding of videotaped naturalistic interactions with a parent. Greater inertia of both negative and positive emotional behaviors predicted the emergence of clinical depression 2.5 years later. The implications of these findings for the understanding of the etiology and early detection of depression are discussed.
\end{abstract}

Keywords: emotional inertia, emotion dynamics, emotion regulation, depression, adolescence, risk-factor

Depression is a devastating mood disorder that afflicts close to one sixth of the population and is considered to be the single most burdensome disease in the world in terms of total disabilityadjusted life years (Kessler \& Wang, 2009). The importance of identifying and understanding the risk factors for depression cannot be overstated. One of the most striking findings from research on the epidemiology of depression is its dramatic rise in incidence during adolescence (Lewinsohn, Rohde, \& Seeley, 1998). Indeed, adolescence is a period marked by significant psychosocial, biological, and cognitive changes that lead to increases in emotionality, greater sensitivity to social interactions, and greater reward seeking and concomitant risk taking (Allen \& Sheeber, 2008). In contrast, the executive and self-regulatory skills to cope with these changes mature more gradually and are not fully developed until

This article was published Online First October 10, 2011.

Peter Kuppens, Department of Psychology, University of Leuven, Leuven, Belgium, and Department of Psychological Sciences, University of Melbourne, Melbourne, Australia; Lisa B. Sheeber, Oregon Research Institute, Eugene, OR; Marie B. H. Yap, Sarah Whittle, Julian G. Simmons, and Nicholas B. Allen, Department of Psychological Sciences, University of Melbourne and Oregon Youth Health Research Centre.

This research was supported by grants from the Colonial Foundation, the National Health and Medical Research Council (NHMRC; Australia; Program Grant 350241), and the Australian Research Council (Discovery Grant DP0878136). The first author is a Postdoctoral Research Fellow with the Fund for Scientific Research-Flanders, and is supported by KULeuven Research Council Grants GOA/05/04 and OT/11/031.

Correspondence concerning this article should be addressed to Nicholas B. Allen, Department of Psychological Sciences and Orygen Youth Health Research Centre, University of Melbourne, VIC 3010 Australia. E-mail: nba@unimelb.edu.au the third decade of life (Steinberg, 2005). How adolescents manage and regulate their intensified emotional life is a developmental challenge that is hypothesized to play a key part in the increased vulnerability for depression observed during this phase of the life span (Allen \& Sheeber, 2008).

\section{Emotional Inertia}

How people respond to and regulate emotional events is directly reflected in the patterns and regularities with which their emotions change across time (Larsen, 2000). While tonic levels of emotion and mood are indisputably important for adjustment, one of the most consequential findings from research on the dynamics of emotions is indeed that how emotions change across time is intricately tied to people's well-being and maladjustment (e.g., Ebner-Priemer, Eid, Kleindienst, Stabenow, \& Trull, 2009; Kuppens, Oravecz, \& Tuerlinckx, 2010b).

Recently, we examined the concept of emotional inertia as a central feature of such emotion dynamics (Kuppens, Allen, \& Sheeber, 2010a). Emotional inertia was first introduced by Suls, Green, and Hillis (1998) as a particular dynamical characteristic of people's emotional fluctuations. In essence, it reflects the extent that one's current emotional state is predictable from one's prior emotional state. High emotional inertia thus reflects that a person's emotional fluctuations show a high degree of momentum, with current emotional states being heavily influenced by previous states. Low emotional inertia, on the other hand, reflects the opposite, and indicates that current feelings are less predictable from previous feelings. Importantly, emotional inertia is distinct from emotional variability, which reflects the range of fluctuations in emotion (i.e., the average deviation form one's own average emotional state). A person's emotions can be highly variable and 
highly inert (e.g., slow but large mood fluctuations), highly variable and low in inertia (e.g., frequent large mood shifts), low in variability and high in inertia (e.g., slow small mood shifts), or low in both variability and inertia (e.g., frequent but small mood shifts; see, e.g., Ebner-Priemer et al., 2009; Jahng, Wood, \& Trull, 2008; Trull et al., 2008). In a way, inertia reflects the speed of change while variability reflects the extent of change.

\section{Emotional Inertia and Depression}

The fact that our emotions change lies at the very heart of their adaptive nature, allowing the individual to be alerted and motivated to respond to potential threats and challenges encountered during daily life. Indeed, emotional responses afford an individual the flexibility to be in tune with and respond to ongoing events, and such flexibility is thought to be crucial for psychological health (Kashdan \& Rottenberg, 2010). High levels of emotional inertia, however, may be a sign that emotional responses have become decoupled from environmental or psychological demands and, thus, may be indicative of maladjustment. In particular, high emotional inertia may reflect that a person's emotions are less responsive to external (e.g., changes in the environment) or internal influences (e.g., regulation efforts), and as such capture both the dampened emotional reactivity (e.g., Bylsma, Morris, \& Rottenberg, 2008; Rottenberg, 2005) and the impaired emotion regulation skills (e.g., Gross \& Muñoz, 1995) that are documented in mood disorders such as depression.

On the basis of this conceptualization, we recently hypothesized that emotional inertia may be a hallmark feature of the temporal emotion dynamics characteristic of psychological maladjustment (Kuppens et al., 2010a). In two naturalistic studies, we demonstrated that higher levels of emotional inertia for both negative and positive emotions observed in experience sampling (Study 1) and observational data (Study 2) were related to low self-esteem and depression, respectively (Kuppens et al., 2010a). In one of the studies, we showed that the presence of clinical depression in an adolescent sample was associated with increased inertia in observed emotional behavior during interactions with their parents. In a similar vein, other research has shown that internalizing symptoms are associated with behavioral rigidity in parent-child interactions (e.g., Hollenstein, Granic, Stoolmiller, \& Snyder, 2004).

These findings raise a fundamental question about the role of emotional inertia in depression. On the one hand, depression may slow down the way an adolescent's emotions unfold across time, making emotional inertia a concomitant or a consequence of depression. On the other hand, emotional inertia may reflect an early form of emotional dysregulation that creates vulnerability to disorder. An emotional life characterized by high levels of inertia may adversely affect emotional functioning, due to the decoupling of emotions from their adaptive function of being responsive to environmental and internal changes. The hypothesis that emotional inertia reflects a risk factor for depression is consistent with current knowledge regarding other predictors of depression. Biologically, risk for depression involves genetic factors related to impairments in both extinction circuits for negative affect and regulatory networks mediating emotional reactivity (Pezawas et al., 2005). Cognitively, rumination (i.e., the tendency to brood over negative thoughts), which could be a cognitive mechanism under- lying emotional inertia, is seen as a major risk factor for depression (Nolen-Hoeksema, Wisco, \& Lyubomirski, 2008; Spasojevic \& Alloy, 2001). Moreover, Coifman and Bonanno (2010) recently found that low context sensitivity, or the incapacity to shift emotions according to changing context, differentiated people that were able to recover after bereavement from people that developed depressive symptoms. These data suggest that emotional inertia may reflect an early risk factor for the development of depressive disorder. The cross-sectional nature of our earlier study, however, precluded examination of the hypothesis that emotional inertia precedes and predicts the onset of the depression.

\section{This Study}

The aim of this article is to determine whether emotional inertia prospectively predicts the first onset of depressive disorder in adolescence. As well as replicating our previous results with a different sample, such a finding would identify emotional inertia as a risk factor for the development of clinical depression. It is important to establish that emotional inertia predicts the onset of depression above other known risk factors in order to reduce the possibility that any found prospective relationship is due to relationships with a third variable. We therefore examined the prospective relationship between emotional inertia and depression while controlling for other known risk factors for the development of clinical depression, self-reported depressive symptoms (Pine, Cohen, Cohen, \& Brook, 1999), gender (Nolen-Hoeksema \& Girgus, 1994), and also average levels of emotionality (Morris, Bylsma, \& Rottenberg, 2009). If emotional inertia is able to predict the emergence of depression above these factors, it reflects unique, dynamical information on emotional functioning that is not merely reflected in depressive symptoms or average emotion levels and that additionally contributes to gender in affecting vulnerability for depression.

We examined this question using data from a longitudinal study of the development of depression in adolescence, the Orygen Adolescent Development Study (Whittle et al., 2008; Yap et al., 2008). Emotional inertia was assessed based on videotaped interactions between adolescents and a parent (following a method similar to that described in Kuppens et al., 2010a), and was used to prospectively predict the emergence of case-level depression about 2.5 years later.

\section{Method}

\section{Participants}

Participants were recruited using a two-gate procedure. The first phase consisted of a large $(N=2,479)$, representative classroombased screening of Year Six primary school students (age range: 9-12 years) using the Early Adolescent Temperament Questionnaire-Revised (EATQ-R; Ellis \& Rothbart, 2001). The EATQ-R measures aspects of temperament related to emotionality, reactivity, and self-regulation (Putnam, Ellis, \& Rothbart, 2001). Given that the main aim of the screening procedure was to provide a risk-enriched sample for the more intensive assessment phase, EATQ-R scores were used to select a sample that was representative of scores across the full range of the temperament dimensions. Selection was based on scores on Negative Emotionality 
(NEM; measuring the tendency for frustration) and Effortful Control (EC; measuring the capacity for attention and self-regulation; Putnam et al., 2001) dimensions, given their hypothesized role as correlates and risk factors for emotional and behavioral disorders (Rothbart \& Bates, 2006; Yap et al., 2011). Accordingly, equal numbers of male and female students were selected from each of the following ranges of scores on each dimension: $0-1,1-2,2-2.5$, and greater than $2.5 S D$ above and below the mean. This produced a risk-enriched sample for the subsequent assessment phase that showed a normal distribution of EC and NEM, albeit one with larger variance than an unselected sample. The sample $(n=245)$ was subsequently administered a semistructured clinical interview for $D S M-I V$ Axis-I disorders (Schedule for Affective Disorders and Schizophrenia for School-Age Children-Present and Lifetime version; K-SADS-PL, Kaufman et al., 1997). Of this sample, 198 families agreed to participate in a laboratory session at Time 1 (which took place on average 10 months after initial screening but within weeks after the clinical interview; average age at $\mathrm{T} 1=$ 12.47 years, range $11.38-13.61$ ) and also completed a self-report measure of depressive symptoms. At Time 2 (approx. 31 months after T1), a total of 179 adolescents ( 89 girls; average age $=15.04$ years, range 13.73-16.19) participated in a follow-up K-SADS diagnostic interview at home. From this sample, data on all variables was available for 167 participants.

At T1, two participants met diagnostic criteria for past depressive disorder and none for current depressive disorder. The two participants with a history of depression at $\mathrm{T} 1$ were removed from the prospective analyses to allow the prediction of the first onset of depression, leaving a final sample of 165 participants. At T2, a total of 22 participants (15 female) were diagnosed with major depressive disorder.

\section{Materials}

Diagnostic interview. The K-SADS-PL (Kaufman et al., 1997) is a semistructured diagnostic interview assessing current and past symptoms and diagnoses of Axis I disorders in youths aged 6 to 18 years. The K-SADS-PL has demonstrated good psychometric properties, and comparison with other child diagnostic interviews suggests good diagnostic validity (Kaufman et al., 1997). Recordings of $25 \%$ of all K-SADS-PL interviews at T2 were rerated by a second coder. The kappa coefficient for diagnosis-level agreement was 0.84, suggesting acceptably high interrater reliability.

Depressive symptoms. Self-reported depressive symptoms at T1 were assessed with the Center for Epidemiological Studies
Depression scale (CESD; Radloff, 1977), a scale designed to assess depressive symptoms in the general population. The CESD consists of 20 items to be rated on a 4-point scale from 0 (rarely or none of the time) to 3 (most or all of the time). The average total score on the CESD for the final sample was $11.54(S D=9.86$, min. $=0$, max. $=55$; Table 1$)$. This average score reflects few or mild depressive symptoms and is comparable to other reported scores of community adolescent samples (e.g., Radloff, 1991).

\section{Procedure}

At Time 1, the adolescent and one parent in consenting families were invited to participate in two 20-min family interaction tasks in the laboratory (the participating parent being the mother in 135 or $82 \%$ of the families). The first task focused on planning a pleasant family activity, and the second focused on resolving an area of conflict. As Kuppens et al. (2010a) demonstrated that differences in emotional inertia between depressed and nondepressed adolescents emerge particularly in the latter, more emotionally taxing type of interaction, only data from this task were used in the current study. The interaction task was video-recorded for subsequent behavioral coding using the Living in Family Environments coding system (LIFE; Hops, Biglan, Tolman, Arthur, \& Longoria, 1995). The validity of the LIFE system to measure affective behavior in families of adolescents has been established in numerous studies (e.g., Katz \& Hunter, 2007; Sheeber, Davis, Leve, Hops, \& Tildesley, 2007). Extensively trained observers, blind to diagnostic status and hypotheses, coded the adolescents' and parents' nonverbal affect and verbal content. Three nonoverlapping constructs indicating angry, dysphoric, and happy behavior were derived from the coding system. Angry behavior included aggressive or contemptuous nonverbal behavior and cruel or provoking statements. Dysphoric behavior was defined by sad nonverbal behavior or statements. Happy behavior reflected happy nonverbal behavior or statements. Approximately $20 \%$ of the interactions were coded by a second observer to provide an estimate of observer agreement. Kappa coefficients were $.77, .68$, and .89 for the respective composite constructs. The procedure yielded second-by-second binary time-series information on the emotional (happy, angry, dysphoric) behavior of adolescents during a real-life interaction with one of their parents, and was used to derive a measure of emotional inertia. They also completed a number of self-report instruments, including the CESD. At T2, participants were administered the K-SADS diagnostic interview (Kaufman et al., 1997) along with a number of other assessments.

Table 1

Means, Standard Deviations, and Intercorrelations Between T2 Depressive Status $(1=$ Depressed; $0=$ Nondepressed $)$, Gender $(1=$ Female; $0=$ Male), CESD Scores at T1, and Mean Observed Behavior for Each Emotion During the Interaction $(\mathrm{N}=165)$

\begin{tabular}{|c|c|c|c|c|c|c|c|}
\hline & $M$ & $S D$ & 1 & 2 & 3 & 4 & 5 \\
\hline 1. T2 depressive status & - & - & & & & & \\
\hline 2. Gender & - & - & $.14^{\dagger}$ & & & & \\
\hline 3. T1 CESD & 11.54 & 9.86 & .11 & -.07 & & & \\
\hline 4. Average Angry behavior & .21 & .19 & $.19^{*}$ & $.15^{\dagger}$ & .10 & & \\
\hline 5. Average Dysphoric behavior & .17 & .14 & $.14^{\dagger}$ & -.06 & .01 & $-.25^{\text {*** }}$ & \\
\hline 6. Average Happy behavior & .30 & .16 & $-.17^{*}$ & -.06 & -.06 & $-.58^{* * *}$ & $-.40^{* * * *}$ \\
\hline
\end{tabular}

${ }^{\dagger} p<.10 . \quad{ }^{*} p<.05 . \quad{ }^{* * *} p<.01 .{ }^{* * * *} p<.001$. 


\section{Data Analyses}

The data analyses followed a two-step procedure (for a similar approach, see, e.g., Caminis, Henrich, Ruchkin, Schwab-Stone, and Martin, 2007). ${ }^{1}$ In the first step, estimates of emotional inertia were obtained from two-level (seconds nested in persons) logistic multilevel analyses performed separately for each emotion on the behavioral observation data from the interaction task. As in Kuppens et al. (2010a), emotional inertia was operationalized as the autocorrelation of emotional behavior throughout the interactions (e.g., the degree to which an emotional behavior is predicted by that same behavior at a previous time moment). Specifically, for each emotion, the adolescents' behavior (e.g., angry behavior) at time $t$ in the interaction task was predicted by that same behavior at time $t-5 \mathrm{~s}^{2}$ at level 1 of the model (the lagged predictors being group-mean centered, Enders \& Tofighi, 2007). At level 2 of the models, person-specific (random) intercept and slope values were estimated (intercept and slope estimates were allowed to covary across participants; the covariance estimates were $-.09,-.05$, and -.50 for angry, dysphoric, and happy behavior). The personspecific intercept values reflect individual differences in the average level of the emotional behavior throughout the interaction, while, the slope values reflect individual differences in autocorrelation or inertia of the emotional behavior (e.g., how much a behavior is predicted by that same behavior 5 seconds ago).

In the next step, these estimates were used to predict depressive status at T2 in logistic regression analyses, controlling for gender and CESD scores at T1. Logistic analyses were performed in which T2 depressive status was predicted by the intercept estimates of each behavior (e.g., by the average level of the behavior during interaction; Models 1), by the slope estimates of each behavior (e.g., by the autocorrelation/inertia of the behavior during interaction; Models 2), and by both (Models 3). The results of these analyses indicate the extent to which average emotional behavior and emotional inertia, separately or combined, prospectively predict the presence of depressive disorder approximately 2.5 years later, beyond the contribution of initial depressive symptoms and gender. As noted, all analyses included the 165 participants without prior depressive disorder at T1.

\section{Results}

Table 1 reports the means, standard deviations, and intercorrelations between depressive status, gender, CESD scores at T1, and mean observed behavior for each emotion during the interaction. The results of the main analyses are presented in Table 2. Both depressive symptoms and female gender marginally predicted depression in most analyses. Average levels of anger and happy behavior also contributed positively and negatively, respectively, to the prediction of depression above these risk factors (see Models 1).

Of direct interest to the current research question, the results showed that inertia for all three studied emotions was a significant positive predictor of depressive status at $\mathrm{T} 2$. These results demonstrate that higher levels of emotional inertia at T1 uniquely predicted the emergence of depression at T2 above the included risk factors. Moreover, when both average behavior and inertia were entered simultaneously (Models 3), the results showed that emotional inertia remained a significant $(p=.051$ in the case of
Table 2

Results From Logistic Regression Analyses Predicting Depressive Status at T2 by Either (Model 1 and Model 2) or Both (Model 3) Average Emotion Behavior and Emotional Inertia Observed at T1, Controlling for T1 Depressive Symptoms and Gender $(1=$ Female; $0=$ Male $)(\mathrm{N}=165)$

\begin{tabular}{|c|c|c|c|c|}
\hline \multicolumn{5}{|c|}{ Behavior Model } \\
\hline Predictors & $B$ & $S E$ & Odds-ratio & $p$ \\
\hline \multicolumn{5}{|l|}{ ANGER } \\
\hline \multicolumn{5}{|l|}{ Model 1} \\
\hline T1 depressive symptoms & .028 & .022 & 1.028 & .209 \\
\hline Gender & .791 & .537 & 2.206 & .141 \\
\hline Average anger & .392 & .175 & 1.408 & .025 \\
\hline \multicolumn{5}{|l|}{ Model 2} \\
\hline T1 depressive symptoms & .037 & .022 & 1.038 & .092 \\
\hline Gender & 1.033 & .537 & 2.809 & .055 \\
\hline Inertia anger & .790 & .381 & 2.204 & .038 \\
\hline \multicolumn{5}{|l|}{ Model 3} \\
\hline T1 depressive symptoms & .031 & .023 & 1.031 & .172 \\
\hline Gender & .814 & .548 & 2.257 & .138 \\
\hline Average anger & .368 & .172 & 1.445 & .032 \\
\hline Inertia anger & .745 & .382 & 2.106 & .051 \\
\hline \multicolumn{5}{|l|}{ DYSPHORIA } \\
\hline \multicolumn{5}{|l|}{ Model 1} \\
\hline T1 depressive symptoms & .034 & .021 & 1.034 & .114 \\
\hline Gender & 1.027 & .534 & 2.792 & .054 \\
\hline Average dysphoria & .336 & .242 & 1.399 & .165 \\
\hline \multicolumn{5}{|l|}{ Model 2} \\
\hline T1 depressive symptoms & .039 & 0.22 & 1.039 & .075 \\
\hline Gender & .867 & .537 & 2.379 & .107 \\
\hline Inertia dysphoria & .867 & .401 & 2.379 & .030 \\
\hline \multicolumn{5}{|l|}{ Model 3} \\
\hline T1 depressive symptoms & .039 & .022 & 1.040 & .076 \\
\hline Gender & .922 & .546 & 2.515 & .091 \\
\hline Average dysphoria & .344 & .237 & 1.411 & .146 \\
\hline Inertia dysphoria & .895 & .404 & 2.447 & .027 \\
\hline \multicolumn{5}{|l|}{ HAPPY } \\
\hline \multicolumn{5}{|l|}{ Model 1} \\
\hline T1 depressive symptoms & .032 & .022 & 1.033 & .140 \\
\hline Gender & .881 & .536 & 2.415 & .100 \\
\hline Average happy & -.517 & .227 & 0.596 & .023 \\
\hline \multicolumn{5}{|l|}{ Model 2} \\
\hline T1 depressive symptoms & .036 & .023 & 1.037 & .109 \\
\hline Gender & .902 & .550 & 2.465 & .101 \\
\hline Inertia happy & 1.176 & .348 & 3.240 & .001 \\
\hline \multicolumn{5}{|l|}{ Model 3} \\
\hline T1 depressive symptoms & .037 & .023 & 1.038 & .105 \\
\hline Gender & .927 & .555 & 2.527 & .095 \\
\hline Average happy & .150 & .361 & 1.162 & .678 \\
\hline Inertia happy & 1.343 & .536 & 3.830 & .012 \\
\hline
\end{tabular}

\footnotetext{
${ }^{1}$ We thank two anonymous reviewers for suggesting this data analytic plan.

${ }^{2}$ Consistent with Kuppens et al. (2010a), a 5-s lag was chosen because it was considered a meaningful time interval to study emotional change in the context of a live ongoing social interaction. To check against the possibility of spurious findings obtained with just that interval, all analyses reported in the article were also run with a 10-s lag, supporting identical conclusions (one minor exception was that the predictive effect of dysphoric inertia for $\mathrm{T} 2$ depression became marginally significant, with $p=$ $.11)$.
} 
anger) predictor of depressive status above and beyond average behavior. For two of the three studied emotions, emotional inertia even emerged as the only significant predictor of depression, above average levels, depressive symptoms, and gender. ${ }^{3}$

Because in this study emotional inertia was measured in the context of a dyadic interaction between adolescent and parent, the interpretation of the results so far may be ambiguous with respect to the extent that observed inertia indeed reflects an individual internal process, versus being a property of the external interaction or interaction partner in which it is observed (e.g., inertia could result from a particular parental interaction style such as being less expressive in families with depressed adolescents, instead of characterizing the emotion dynamics of the adolescent). Therefore, to control for potential influence of the dyadic interaction, we reestimated the inertia/autocorrelation parameters, based on logistic multilevel models, which though similar to those described above also included the corresponding parental behavior at $t-5 \mathrm{~s}$ as a predictor at level 1 (e.g., adolescent angry behavior was predicted by adolescent angry behavior at $t-5 \mathrm{~s}$ and parent angry behavior at $t-5 \mathrm{~s})$. The resulting inertia estimates reflect emotional inertia after controlling for potential influence of (corresponding) parental behavior, and may, therefore, reflect a purer measure of endogenously determined inertia. In the second step, these estimates were again entered in a logistic regression analyses predicting $\mathrm{T} 2$ depressive status, controlling for CESD and gender. If the predictive value of these inertia estimates (in which the corresponding parental influence on the behavior is controlled for) remains, this is a strong indicator that inertia reflects an internal process instead of a property caused by the external interaction in the prediction of depression. The results indeed supported this conclusion. Based on inertia estimates that controlled for the corresponding parental behavior, inertia for angry $(B=.761, S E=.386, p=.048$, Odds-Ratio $(O R)=2.141)$, dysphoric $(B=.824, S E=.401, p=$ $.040, O R=2.279)$ and happy behavior $(B=1.111, S E=.345$, $p=.001, O R=3.036)$ still predicted the onset of $\mathrm{T} 2$ depression, independent of gender and depressive status. The same analyses additionally including average emotional behavior (intercept) yielded similar results (angry: $B=.715, S E=.39, p=.064$, $O R=2.043$; dysphoric: $B=.855, S E=.406, p=.035, O R=$ 2.351; happy: $B=1.169, S E=.513, p=.023, O R=3.218$ ).

\section{Discussion}

The findings from this study replicate our previous findings (Kuppens et al., 2010a) in a third, independent sample, providing strong additional support for the importance of emotional inertia as a hallmark feature of emotion dynamics in emotional maladjustment. More importantly, they establish emotional inertia as a prospective risk factor for the onset of depressive disorder in adolescence, a life phase that involves a particular vulnerability for the development of mood disorder.

The fact that emotional inertia may be a particularly strong risk factor or predictor of depression is suggested by the fact that it was a significant predictor above and beyond two other known risk factors-extent of depressive symptoms and gender-and above the predictive value of average levels of emotional behavior observed during the interactions. These results add to the robustness of findings regarding the relationship between inertia and depression, and strengthen the conclusion that emotional inertia is not merely the result of depressed mood and related symptoms, but is independent of it in its predictive role for depression. They also underline that emotional inertia reflects dynamical processes that create vulnerability to depression, unique from that conferred by average levels of affective behavior.

This leads to the question: What is it, then, in emotional inertia that makes people susceptible to depression? First of all, the presented results support the idea that emotional inertia reflects a characteristic style of emotional functioning in the individual that is predictive of depression. Indeed, the results from the additional analyses demonstrated that emotional inertia remained predictive even after parental behavior was controlled for in the inertia estimates. This suggests that emotional inertia as a predictor of depression may be viewed as a property that characterizes the emotion dynamics of the individual, and does not result solely as a function of the behavior of the interaction partner, at least in the context of parent-adolescent interactions. Additional research is needed to confirm that observed inertia is associated with depression after controlling for the interactive behavior of other important interaction partners, like, for instance, an adolescent's peers. Further, it has been hypothesized that emotional inertia reflects a type of emotional functioning characterized by both impaired emotional reactivity and regulation (see, e.g., Kuppens et al., 2010a; Suls et al., 1998). Both biological changes and a host of contextual stressors (involving school, romantic and peer relationships, etc.) make adolescence a period in which many youth are faced with increases in negative emotionality (Larson \& Sheeber, 2008). Speculatively, high emotional inertia may reflect a particular type of coping or emotional style that emerges to deal with this intensified emotional life (although it should be noted that associated phenomena like behavioral rigidity toward parents have also been observed at a younger age in relation to maladjustment; Hollenstein et al., 2004). Such a coping style may involve disconnecting one's emotional responses from their normal contingencies (both internal and external), manifested in higher levels of emotional inertia. In turn, higher levels of emotional inertia may provide further challenges to emotional functioning during this life phase, and may put the adolescent at risk for problematic emotional functioning. At the extreme, the results of such a process may be the development of clinical depression. In this respect, emotional inertia may reflect the emotional counterpart of cognitive rumination, in which thoughts become disconnected from events and regulation and become "stuck" on certain themes, and which is likewise considered a strong risk factor for depression (Joormann, 2010; Nolen-Hoeksema et al., 2008). Further study is needed, however, to clarify the precise determinants and the developmental patterns associated with emotional inertia and how it relates to other dynamical characteristics such as variability in emotional intensity.

Similar to Kuppens et al. (2010a), our results showed that inertia for both negative and positive emotions was related to depression. Depression is indeed thought to be characterized by disruptions in the functioning of both negative and positive affect (Clark \& Watson, 1991). Speculatively, the higher inertia observed for

\footnotetext{
${ }^{3}$ Supplementary analyses (additionally including an interaction term between gender and inertia estimates) showed that the predictive effect of inertia for all three emotions was not moderated by gender $(p s>.50)$.
} 
happy behavior may reflect positive affectivity becoming relatively decoupled from reward (its primary elicitor), which is consistent with the notion of low reward sensitivity as a key aspect of depression (Carver, Johnson, \& Joormann, 2008).

Although the present study has a number of important strengths, such as its prospective nature and the ecological validity of the interactions, it is not without limitations. Only behavioral indicators of inertia were used. Although previous research has shown that inertia in self-reported emotional experience is related to maladjustment as well (Koval \& Kuppens, 2011; Kuppens et al., 2010a; Suls et al., 1998), the present study can only speak to the behavioral component of emotion. The interaction task did also not allow us to examine inertia in noninterpersonal contexts, which could have provided a more direct test of the endogenous nature of emotional inertia. Finally, given the use of a selected sample and the low number of participants diagnosed with depression at T2, future research will be desirable in order to examine the replicability and generalizability of the findings.

Finally, our findings suggest the possibility that early detection of emotional inertia may help to identify youth at risk for mood disorder. Importantly, they also highlight the need for more research into the exact mechanisms that underlie inertia and the mechanisms by which emotional inertia drives the escalation into depressive disorder. Such research will not only help to elucidate the etiology of depression during adolescence, but also provide information relevant to early intervention.

\section{References}

Allen, N. B., \& Sheeber, L. B. (2008). The importance of affective development for the emergence of depressive disorder during adolescence. In N. B. Allen \& L. B. Sheeber (Eds.), Adolescent emotional development and the emergence of depressive disorders (pp. 1-10). Cambridge, UK: Cambridge University Press. doi:10.1017/ CBO9780511551963.001

Bylsma, L. M., Morris, B. H., \& Rottenberg, J. (2008). A meta-analysis of emotional reactivity in major depressive disorder. Clinical Psychology Review, 28, 676-691. doi:10.1016/j.cpr.2007.10.001

Caminis, A., Henrich, C., Ruchkin, V., Schwab-Stone, M., \& Martin, A. (2007). Psychosocial predictors of sexual initiation and high-risk sexual behaviors in early adolescence. Child Adolescent Psychiatry \& Mental Health, 1, 14. doi:10.1186/1753-2000-1-14

Carver, C. S., Johnston, S. L., \& Joormann, J. (2008). Serotonergic function, two-mode models of self-regulation, and vulnerability to depression: What depression has in common with impulsive aggression. Psychological Bulletin, 134, 912-943. doi:10.1037/a0013740

Clark, L. A., \& Watson, D. (1991). Tripartite model of anxiety and depression: Psychometric evidence and taxonomic implications. Journal of Abnormal Psychology, 100, 316-336. doi:10.1037/0021-843X .100 .3 .316

Coifman, K. G., \& Bonanno, G. A. (2010). When distress does not become depression: Emotion context sensitivity and adjustment to bereavement. Journal of Abnormal Psychology, 119, 479-490. doi:10.1037/a0020113

Ebner-Priemer, U. W., Eid, M., Kleindienst, N., Stabenow, S., \& Trull, T. J. (2009). Analytic strategies for understanding affective (in)stability and other dynamic processes in psychopathology. Journal of Abnormal Psychology, 188, 195-202. doi:10.1037/a0014868

Ellis, L. K., \& Rothbart, M. K. (2001, April). Revision of the Early Adolescent Temperament Questionnaire. Paper presented at the Biennial Meeting of the Society for Research in Child Development, Minneapolis, MN.

Enders, C. K., \& Tofighi, D. (2007). Centering predictor variables in cross-sectional multilevel models: A new look at an old issue. Psychological Methods, 12, 121-138. doi:10.1037/1082-989X.12.2.121

Gross, J. J., \& Muñoz, R. F. (1995). Emotion regulation and mental health. Clinical Psychology: Science and Practice, 2, 151-164. doi:10.1111/ j.1468-2850.1995.tb00036.x

Hollenstein, T., Granic, I., Stoolmiller, M., \& Snyder, J. (2004). Rigidity in parent-child interactions and the development of externalizing and internalizing behavior in early childhood. Journal of Abnormal Child Psychology, 32, 595-607. doi:10.1023/B:JACP.0000047209.37650.41

Hops, H., Biglan, A., Tolman, A., Arthur, J., \& Longoria, N. (1995). Living in Family Environments (LIFE) coding system: Manual for coders (Revised). Eugene, OR: Oregon Research Institute.

Jahng, S., Wood, P. K., \& Trull, T. J. (2008). Analysis of affective instability in EMA: Indices using successive difference and group comparison via multilevel modeling. Psychological Methods, 13, 345-375. doi: $10.1037 / \mathrm{a} 0014173$

Joormann, J. (2010). Cognitive inhibition and emotion regulation in depression. Current Directions in Psychological Science, 19, 161-166. doi:10.1177/0963721410370293

Kashdan, T. B., \& Rottenberg, J. (2010). Psychological flexibility as a fundamental aspect of health. Clinical Psychology Review, 30, 865-878. doi:10.1016/j.cpr.2010.03.001

Katz, L. F., \& Hunter, E. C. (2007). Maternal meta-emotion philosophy and adolescent depressive symptomatology. Social Development, 16, 343-360. doi:10.1111/j.1467-9507.2007.00388.x

Kaufman, J., Birmaher, B., Brent, D., Rao, U., Flynn, C., Moreci, P., ... Ryan, N. (1997). Schedule for Affective Disorders and Schizophrenia for School-Age Children-Present and Lifetime version (KSADS-PL): Initial reliability and validity data. Journal of the American Academy of Child and Adolescent Psychiatry, 36, 980-988. doi: 10.1097/00004583-199707000-00021

Kessler, R. C., \& Wang, P. S. (2009). Epidemiology of depression. In I. H. Gotlib \& C. L. Hammen (Eds.), Handbook of depression, 2nd ed. (pp. 5-22). New York, NY: Guilford Press.

Koval, P., \& Kuppens, P. (2011). Changing emotion dynamics: Individual differences in the effect of social stress on emotional inertia. Manuscript submitted for publication.

Kuppens, P., Allen, N. B., \& Sheeber, L. B. (2010a). Emotional inertia and psychological maladjustment. Psychological Science, 21, 984-991. doi: 10.1177/0956797610372634

Kuppens, P., Oravecz, Z., \& Tuerlinckx, F. (2010b). Feelings change: Accounting for individual differences in the temporal dynamics of affect. Journal of Personality and Social Psychology, 99, 1042-1060. doi:10.1037/a0020962

Larsen, R. J. (2000). Towards a science of mood regulation. Psychological Inquiry, 11, 129-141. doi:10.1207/S15327965PLI1103_01

Larson, R. W., \& Sheeber, L. B. (2008). The daily emotional experience of adolescents: Are adolescents more emotional, why, and how is that related to depression? In N. B. Allen \& L. B. Sheeber (Eds.), Adolescent emotional development and the emergence of depressive disorders (pp. 11-32). Cambridge, UK: Cambridge University Press. doi:10.1017/ CBO9780511551963.002

Lewinsohn, P. M., Rohde, P., \& Seeley, J. R. (1998). Major depressive disorder in older adolescents: Prevalence, risk factors, and clinical implications. Clinical Psychology Review, 17, 765-794. doi:10.1016/ S0272-7358(98)00010-5

Morris, B. H., Bylsma, L. M., \& Rottenberg, J. (2009). Does emotion predict the course of major depressive disorder? A review of prospective studies. British Journal of Clinical Psychology, 48, 255-273. doi: 10.1348/014466508X396549

Nolen-Hoeksema, S., \& Girgus, J. S. (1994). The emergence of gender differences in depression during adolescence. Psychological Bulletin, 115, 424-443. doi:10.1037/0033-2909.115.3.424

Nolen-Hoeksema, S., Wisco, B. E., \& Lyubomirski, S. (2008). Rethinking 
rumination. Perspectives on Psychological Science, 3, 400-424. doi: 10.1111/j.1745-6924.2008.00088.x

Pezawas, L., Meyer-Lindenberg, A., Drabant, E. M., Verchinski, B. A., Munoz, K. E., Kolachana, B. S., ... Hariri, A. R (2005). 5-HTTLPR polymorphism impacts human cingulate-amygdala interactions: A genetic susceptibility mechanism for depression. Nature Neuroscience, 8 , 828-834. doi:10.1038/nn1463

Pine, D. S., Cohen, E., Cohen, P., \& Brook, J. (1999). Adolescent depressive symptoms as predictors of adult depression: Moodiness or mood disorder? American Journal of Psychiatry, 156, 133-135.

Putnam, S. P., Ellis, L. K., \& Rothbart, M. K. (2001). The structure of temperament from infancy through adolescence. In A. Eliasz \& A. Angleitner (Eds.), Advances in research on temperament (pp. 165-182). Lengerich, Germany: Pabst Scientist Publisher.

Radloff, L. S. (1977). The CES-D scale: A self report depression scale for research in the general population. Applied Psychological Measurement, 1, 385-401. doi:10.1177/014662167700100306

Radloff, L. S. (1991). The use of the Center for Epidemiologic Studies Depression Scale in adolescents and young adults. Journal of Youth and Adolescence, 20, 149-166. doi:10.1007/BF01537606

Rothbart, M. K., \& Bates, J. E. (2006). Temperament. In N. Eisenberg, W. Damon, \& R. M. Lerner (Eds.), Handbook of child psychology: Vol. 3. Social, emotional, and personality development (pp. 99-166). Hoboken, NJ: Wiley.

Rottenberg, J. (2005). Mood and emotion in major depression. Current Directions in Psychological Science, 14, 167-170. doi:10.1111/j.09637214.2005.00354.x

Sheeber, L. B., Davis, B., Leve, C., Hops, H., \& Tildesley, E. (2007). Adolescents' relationships with their mothers and fathers: Associations with depressive disorder and subdiagnostic symptomatology. Journal of Abnormal Psychology, 116, 144-154. doi:10.1037/0021-843X.116 .1 .144
Spasojevic, J., \& Alloy, L. B. (2001). Rumination as a common mechanism relating depressive risk factors to depression. Emotion, 1, 25-37. doi: 10.1037/1528-3542.1.1.25

Steinberg, L. (2005). Cognitive and affective development in adolescence. Trends in Cognitive Sciences, 9, 69-74. doi:10.1016/j.tics.2004.12.005

Suls, J., Green, P., \& Hillis, S. (1998). Emotional reactivity to everyday problems, affective inertia, and neuroticism. Personality and Social Psychology Bulletin, 24, 127-136. doi:10.1177/0146167298242002

Trull, T. J., Solhan, M. B., Tragesser, S. L., Jahng, S., Wood, P. K., Piasecki, T. M., \& Watson, D. (2008). Affective instability: Measuring a core feature of borderline personality disorder with ecological momentary assessment. Journal of Abnormal Psychology, 117, 647-661. doi: $10.1037 / \mathrm{a} 0012532$

Whittle, S., Yap, M. B. H., Yücel, M., Fornito, A., Barrett, A., Sheeber, L. \& Allen, N. B. (2008). Prefrontal and amygdala volumes are related to adolescents' affective behaviors during parent adolescent interactions. Proceedings of the National Academy of Sciences of the United States of America, 105, 3652-3657. doi:10.1073/pnas.0709815105

Yap, M. B. H., Allen, N. B., O'Shea, M., Di Parsia, P., Simmons, J. G., \& Sheeber, L. (2011). Early adolescents' temperament, emotion regulation during mother-child interactions and depressive symptomatology. Development and Psychopathology, 23, 267-282. doi:10.1017/ S0954579410000787

Yap, M. B. H., Whittle, S., Yücel, M., Sheeber, L., Pantelis, C., Simmons, J., \& Allen, N. B. (2008). Parenting experiences interact with brain structure to predict depressive symptoms in adolescents. Archives of General Psychiatry, 65, 1377-1385. doi:10.1001/archpsyc.65.12.1377

Received March 11, 2011 Revision received June 9, 2011 Accepted June 14, 2011

\section{Showcase your work in APA's newest database.} PsvcTESTS

Make your tests available to other researchers and students; get wider recognition for your work.

"PsycTESTS is going to be an outstanding resource for psychology," said Ronald F. Levant, PhD. "I was among the first to provide some of my tests and was happy to do so. They will be available for others to use - and will relieve me of the administrative tasks of providing them to individuals."

Visit http://www.apa.org/pubs/databases/psyctests/call-for-tests.aspx to learn more about PsycTESTS and how you can participate.

Questions? Call 1-800-374-2722 or write to tests@apa.org. Not since PsycARTICLES has a database been so eagerly anticipated! 